\title{
Effect of body fat distribution on the transcription response to dietary fat interventions
}

\author{
Marijana Radonjic • Marjan J. van Erk • \\ Wilrike J. Pasman · Heleen M. Wortelboer • \\ Henk F. J. Hendriks · Ben van Ommen
}

Received: 20 March 2009/Accepted: 14 April 2009/Published online: 30 April 2009

(c) The Author(s) 2009. This article is published with open access at Springerlink.com

\begin{abstract}
Combination of decreased energy expenditure and increased food intake results in fat accumulation either in the abdominal site (upper body obesity, UBO) or on the hips (lower body obesity, LBO). In this study, we used microarray gene expression profiling of adipose tissue biopsies to investigate the effect of body fat distribution on the physiological response to two dietary fat interventions. Mildly obese UBO and LBO male subjects $(n=12$, waist-to-hip ratio range $0.93-1.12$ ) were subjected to consumption of diets containing predominantly either long-chain fatty acids (PUFA) or medium-chain fatty acids (MCT). The results revealed (1) a large variation in transcription response to MCT and PUFA diets between UBO and LBO subjects, (2) higher sensitivity of UBO subjects to MCT/PUFA dietary intervention and (3) the upregulation of immune and apoptotic pathways and downregulation of metabolic pathways (oxidative, lipid, carbohydrate and amino acid metabolism) in UBO subjects when consuming MCT compared with PUFA diet. In conclusion, we report that despite the recommendation of MCT-based diet for improving obesity phenotype, this diet may have adverse effect on inflammatory and metabolic status of UBO subjects. The body fat distribution is, therefore, an important parameter to consider when providing personalized dietary recommendation.
\end{abstract}

Keywords Dietary interventions - DNA microarrays . Lower body obesity (LBO) - Upper body obesity (UBO) . Medium-chain fatty acids (MCT) .

Long-chain fatty acids (PUFA)

M. Radonjic $(\bowtie) \cdot$ M. J. van Erk · W. J. Pasman ·

H. M. Wortelboer · H. F. J. Hendriks · B. van Ommen

TNO Quality of Life, Business Unit Biosciences, P.O. Box 360,

3700 AJ Zeist, The Netherlands

e-mail: marijana.radonjic@tno.nl

\section{Introduction}

The principal causes of the increasing obesity problem worldwide are sedentary lifestyles and an increased intake of high-fat, energy-dense diets. Combination of decreased energy expenditure and increased energy or food intake results in fat accumulation either in the abdominal site (upper body obesity, UBO) or on the hips (lower body obesity, LBO), also known as the apple- and pear-shape generalization. Handling of the consumed fatty acids is hypothesized to be different in men with UBO and LBO because of their fat disposition and the routing of the different fatty acids. Upper body obesity also seems to be related to increased metabolic risk [7].

It has been shown that the chain length of dietary fatty acids affects fat absorption, fat metabolism and hepatic gene expression profiles $[4,11,13]$. During the consumption of dietary fats, the first pass of the fatty acids differs: the long-chain fatty acids (PUFA) will be absorbed by the chylomicrons via the lymph and transported through the body to the periphery and the medium-chain fatty acids (MCT) will directly be transported as free fatty acids via the portal vein to the liver $[3,8]$. As a result, PUFA might be disposed to the periphery (organs, muscles or subcutaneous fat) and MCT might easily be disposed centrally. Owing to their rapid breakdown and routing directly to the liver instead of being stored, diet rich in MCT has been advised to people suffering from obesity problems [10, 14].

In this study, we investigated how a difference in body fat distribution affects the physiological response to two dietary fat interventions, namely consumption of a spread containing predominantly PUFA and a spread containing predominantly MCT. We analyzed by microarray gene expression profiling the response of subcutaneous adipose tissue from upper body obese males and LBO males to these two 
different dietary fat interventions. The results suggest that physiological effects of dietary interventions are dependent on body fat distribution. In addition, this study shows that consumption of MCT-rich diet may have adverse effects for upper body obese males, due to the increased inflammation and decreased energy metabolism in adipose tissue.

\section{Methods and materials}

\section{Study design and subjects}

The study was conducted at TNO Quality of Life, where subjects were recruited from a pool of volunteers. After being informed about the study, both verbally and in writing, each subject signed the informed consent form. Twelve healthy male subjects aged between 30 and 60 years and with body mass index (BMI) between 27 and $35 \mathrm{~kg} / \mathrm{m}^{2}$ were included in the study. Subjects were selected so that range of waist-hip ratio (WHR) was as high as possible, in the selected group WHR ranged from 0.93 to 1.12 . Subjects with WHR $<1(n=7)$ were considered LBO and subjects with WHR $>1(n=5)$ upper body obese (UBO) [1].

The study was approved by the Medical Ethics Committee of Tilburg (METOPP) (18 April 2007) and conducted according to the current assembly (52nd) of the Declaration of Helsinki (Edinburgh, Scotland, October 2000) and the ICH Guidelines for Good Clinical Practice (ICH Topic E6, adopted 01-05-1996 and implemented 17-01-1997). The study was designed as a randomized, double-blind, crossover trial and two treatments were supplied for 3 weeks with a wash-out period of 6 weeks in-between. The two dietary interventions consisted of consumption of a spread containing predominantly PUFA (71\% long-chain fatty acids (mainly C18:2), 28\% C16:0 and 1\% fatty acids shorter than C16) and consumption of a spread containing predominantly MCT (65\% C8:0 and C10:0, 29\% C16:0 and 6\% fatty acids longer than $\mathrm{C} 18)$. In both the spreads, a similar percentage of palmitic acid (C16:0: 28-29\%) is present, necessary to make a margarine spreadable.

The treatments were given in random order. Randomization was restricted for age, BMI and WHR. Each day, subjects consumed $60 \mathrm{~g}$ of the spread for 2.5 weeks; during the start of the intervention period subjects only consumed half the amount (so $30 \mathrm{~g}$ of test spread daily) to become habituated to the unusual fatty acids. The test spread replaced their normal spread and oil use. Two portions of $30 \mathrm{~g}$ of spread were consumed with each main meal; portions of $15 \mathrm{~g}$ were provided at the beginning of the intervention period. With the test spread provided, about $50 \%$ of the daily fat intake was of experimental origin. During the complete study period (84 days), no changes were seen in body weight or waist and hip circumferences. Also, the
6 weeks wash-out in-between both test periods did not affect these main characteristics of the study population.

Adipose tissue biopsies were taken at the end of the 3week intervention.

Adipose tissue biopsies

On days 21 and 63, the last day of each supplementation period, biopsies of the subcutaneous fat tissue of the abdomen were taken as follows. The skin was disinfected with alcohol (70\%) and after local anesthesia with lidocaine a fat biopsy was taken by needle aspiration. Part of the fat suspension was immediately frozen in liquid nitrogen and stored at $-80^{\circ} \mathrm{C}$ for RNA isolation. Adipose tissue biopsies were not available from 1 subject, therefore, 11 subjects were included in the microarray analysis.

RNA isolation, labeling and hybridization

RNA was isolated using RNA-Bee (Campro Scientific, Veenendaal, The Netherlands) and glass beads according to the manufacturer's instructions. The integrity of RNA obtained was examined by Agilent Lab-on-a-chip technology using the RNA 6000 Nano LabChip kit and a Bioanalyzer 2100 (both Agilent Technologies, Amstelveen, The Netherlands).

The isolated RNA samples were sent to ServiceXS BV (Leiden, The Netherlands) where they were processed according to Affymetrix protocols. Briefly, RNA concentration was determined by absorbence at $260 \mathrm{~nm}$, and quality and integrity was verified using the Agilent 2100 Bioanalyzer (Agilent Technologies). Next, $2 \mu \mathrm{g}$ of high quality total RNA was used with the Affymetrix Eukaryotic One-Cycle Target Labeling and Control reagents to generate biotin-labeled antisense cRNA. The quality of the cRNA was checked using the Agilent 2100 bioanalyzer.

The labeled cRNA was hybridized to the NuGO Affymetrix Human Genechip NuGO_Hs1a520180 (custom designed by the European Nutrigenomics Organisation NuGO, consisting of 23,941 probesets including 71 control probesets, for details see http://blog.bigcat.unimaas.nl/ martijn/NuGO/). Absolute gene expression values were calculated from the scanned array using the Affymetrix GCOS software. In total, study included 22 microarray hybridizations (two treatments of 11 subjects). The study design including one microarray per subject for each treatment was chosen to maximize the number of biological replicates (6 LBO and 5 UBO subjects) and, thus, power to detect the expression differences.

Data analysis

Quality control and normalization of microarray data was performed using $\mathrm{R} / \mathrm{BioConductor}$ packages through the 
NuGO MadMax pipeline (https://madmax.bioinformatics.nl). Raw signal intensities were normalized using the GCRMA algorithm. The custom CDF file for NuGO_Hs1a520180 (based on Entrez Gene, version 10.0.0; available via http://nugo-r.bioinformatics.nl/NuGO_R.html) was used to re-annotate the probes to new probesets, remove poor quality probes and derive unique signal values for different probesets representing the same gene. This resulted in gene expression values for 16,242 genes with unique identifiers. The gene expression was required to have a value above 10 in at least 1 of 22 samples, resulting in a set of 9,716 genes considered as expressed in adipose tissue. Principal component analysis and analysis of overrepresented Gene Ontology "Biological process" categories were performed using GeneSpring GX 7.3.1 software (Agilent). A paired $t$ test for treatment effect in all subjects and the UBO and LBO subgroups was performed in BRB ArrayTools (software for microarray data analysis developed by Dr. Richard Simon and Amy Peng Lam, http://linus.nci.nih.gov/BRB-ArrayTools.html), using $p$-value of 0.01 as a threshold for significance. The per-subject analysis of over- or under-represented pathways and gene sets upon consuming MCT versus PUFA spread was performed using T-profiler [2]. As input values, $\log _{2}$ ratios of all 9,716 genes were used. Gene ontology (GO, www.geneontology. org), curated gene sets and motif gene sets (Molecular Signatures Database, http://www.broad.mit.edu/gsea/msigdb/ index.jsp) and Kyoto Encyclopedia of Genes and Genomes (KEGG, http://www.genome.jp/kegg/) pathway collections were used as source of queried gene sets. Pathways were considered significant if $E$-value (Bonferroni corrected) was smaller than 0.05. $T$-values resulting from T-profiler analysis were hierarchicaly clustered (Pearson correlation (uncentered), complete linkage) and visualized by HierarchicalClusteringViewer and HeatmapViewer modules within the GenePattern analysis suite $[5,6,12]$. The correlation between WHR and gene expression was analysed in SAS Enterprise guide 4.1 (SAS Institute Inc., Cary, USA). A Pearson correlation coefficient higher than 0.7 or lower then -0.7 , associated with $\mathrm{p}$-value smaller than 0.016 was used as threshold for significance. The gene expression data will be made available through ArrayExpress, a public repository for microarray data (www.ebi.ac.uk/arrayexpress/).

\section{Results}

Mildly obese male subjects with WHR ranging from 0.93 to 1.12 were submitted to MCT- and PUFA-based dietary interventions and the RNA samples obtained from their fat biopsies were hybridized to microarrays (Methods and Materials). Subjects with WHR $<1$ were considered LBO and those with WHR $>1$ upper body obese (UBO) (Table 1).
Table 1 Overview of subject's identification numbers, waist-to-hip ratios (WHR, sorted from lowest to highest) and body fat distribution grouping (either UBO or LBO)

\begin{tabular}{lll}
\hline Subject & WHR & Group \\
\hline S9 & 0.934 & LBO \\
S13 & 0.935 & LBO \\
S4 & 0.971 & LBO \\
S11 & 0.974 & LBO \\
S7 & 0.986 & LBO \\
S8 & 0.990 & LBO \\
S2 & 1.000 & UBO \\
S5 & 1.023 & UBO \\
S3 & 1.030 & UBO \\
S10 & 1.036 & UBO \\
S1 & 1.122 & UBO \\
\hline
\end{tabular}

After preprocessing of the microarray data, normalized expression values were obtained for 9,716 genes expressed in subcutaneous adipose tissue. The global relations among the gene expression signatures of subjects fed two diets were assessed by principal component analysis (PCA) (Fig. 1). This reveals a large inter-subject variation in the transcription response to MCT and PUFA dietary interventions. Moreover, the division of subjects to an UBO and LBO group explains more variance in gene

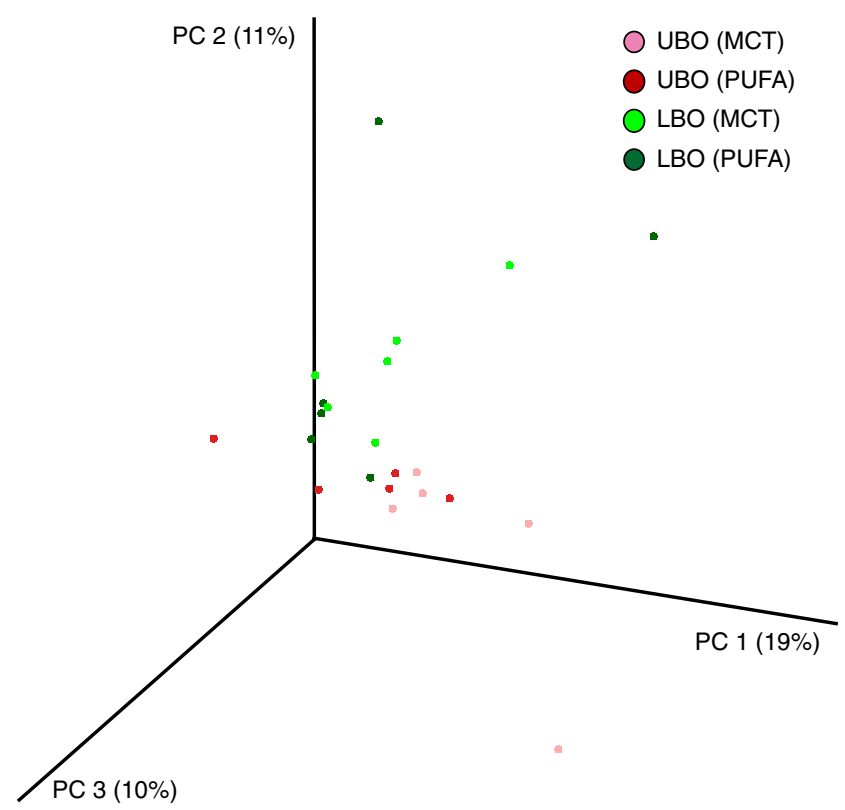

Fig. 1 Principal component analysis of the adipose tissue gene expression. Principal component analysis of the adipose tissue gene expression in 11 subjects, each in two dietary conditions (five UBO and six LBO, each after consumption of both MCT and PUFA spread). There is a large inter-subject variation in gene expression response to MCT and PUFA treatments. The distinction between UBO and LBO groups explains most variance observed in the data (red versus green dots) 
expression than the grouping of subjects according to a diet. This shows that the body fat distribution represents the principal determinant of a diet effect on the adipose tissue gene expression. The observed relevance of body fat distribution for the effects of dietary fat interventions underscores different physiological characteristics of UBO and LBO subjects and suggests that this phenotypic property should be considered when assessing MCT and PUFA diet effects.

The distinct transcriptional response of UBO and LBO subjects was supported by statistical analysis of differentially expressed genes between MCT- and PUFA-based dietary interventions. To investigate the overall effects of the MCT and PUFA diets, a paired (per subject) $t$ test comparing two interventions (MCT vs. PUFA) was performed in (1) all subjects and (2) separately in UBO and LBO groups. Applying the statistical cutoff of $p$-value $<0.01,179$ genes were identified as differentially expressed among all subjects, whereas 239 and 73 genes were identified as differentially expressed in UBO and LBO groups, respectively. Despite the decreased sample number when analysis was performed separately for UBO and LBO groups, we were able to identify a larger number of differentially expressed genes in the UBO group than when all subjects were analysed. This confirms the large heterogeneity of inter-subject responses to two dietary interventions observed by PCA and the dependence of this response on the subject's body fat distribution. In addition, three times larger number of differentially expressed genes in UBO compared to LBO group shows that UBO subjects are more sensitive than LBO subjects to MCT versus PUFA dietary intervention.

The effects of body fat distribution on the changes in biological processes upon consuming MCT and PUFA spread were analyzed by T-profiler [2]. This method enables subject-specific identification of biological processes that are significantly induced or repressed by dietary interventions. To increase the robustness of the analysis, four collections of gene sets associated with various biological processes were used for analysis as described in "Methods and Materials" section. The resulting T-scores represent relative up- and down-regulation of pathways in MCT versus PUFA comparison. In total, 547 pathways were identified as significantly regulated in at least one of 11 subjects (Fig. 2). The T-scores were hierarchically clustered, resulting in aggregation of similarly regulated pathways. In addition, hierarchical clustering of subjects based on their pathway profiles (Fig. 2a) showed correlation of subject's responses to MCT compared to PUFA. In agreement with PCA and statistical analysis, subjects with tendency to LBO (WHR 0.93-0.97, subjects S9, S13, S4 and S11) reacted differently to the dietary fat intervention than the subjects with tendency to UBO (WHR 0.99-1.12, subjects S7, S8, S3, S5, S10, S2 and S1). Focusing on particular sub-clusters revealed that the subjects with higher WHR have higher expression of the apoptotic, inflammatory and immune response pathways (Fig. 2b) and lower expression of mitochondrial, oxidative, fatty acid and amino acid metabolic pathways (Fig. 2c) than the subjects with lower WHR when they are consuming MCT compared to PUFA spread. These findings underscore the impact of the body fat distribution on the important factors in obesity-associated disorders, such as inflammation and energy metabolism of adipose tissue. Observed differential regulation of these processes by MCT and PUFA diets in UBO and LBO subjects imply that harmonization of a diet composition with the body fat distribution profile is necessary for improving subject's obesity status by dietary intervention.

The dependence of biological effects of two diets on the subject's body fat distribution was further investigated by correlation of individual gene expression changes $\left(\log _{2}\right.$ (MCT/PUFA $\left.)\right)$ to the WHR values. Applying the significance threshold of $(r)>0.7$ or $<-0.7$ (Pearson correlation) and $p$-value $<0.016,44$ genes were found to be positively correlated and 165 negatively correlated with WHR (data not shown). The correlation of gene expression with WHR identifies genes which expression in response to MCT versus PUFA dietary intervention can be predicted by subject's body fat distribution. Positive correlation implies that in MCT/PUFA comparison, the gene will be upregulated in UBO subjects and downregulated in LBO subjects. Conversely, negative correlation means that in the MCT/ PUFA comparison, given gene will be downregulated in UBO but upregulated in LBO subjects. Consistent with the pathway analysis results obtained by T-profiler, biological functions of genes with significant positive correlation between $\log _{2}$ (MCT/PUFA) ratio and WHR are involved in immune response and mitochondrial apoptosis, while genes with negative correlation of $\log _{2}$ (MCT/PUFA) ratio and WHR are implicated in metabolic and oxidative processes (Fig. 3a, b). As an increased inflammation and decreased energy metabolism in adipose tissue are considered to have negative effect on obesity-associated disorders, our results suggest that consumption of MCT-instead of PUFA-rich diet may have adverse effects in UBO subjects, while it may be beneficial to LBO subjects.

Examples of genes that have MCT versus PUFA expression ratio significantly correlated to subject's WHR values are MICB (MHC class I polypeptide-related sequence B) (positive correlation) and $A D I P O Q$ (adiponectin) (negative correlation). MICB is a stress-induced protein involved in inflammatory/immune response and low adiponectin expression represents known marker of obesity-associated disorders. MICB MCT/PUFA expression ratio increases in subjects with higher WHR (UBO) 
A

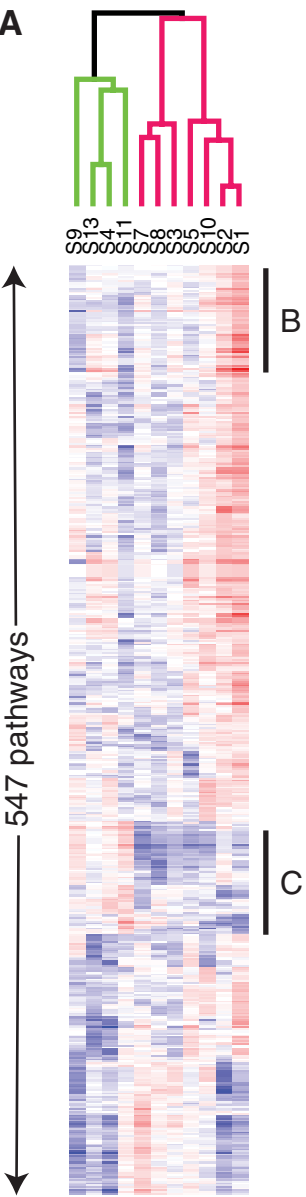

B

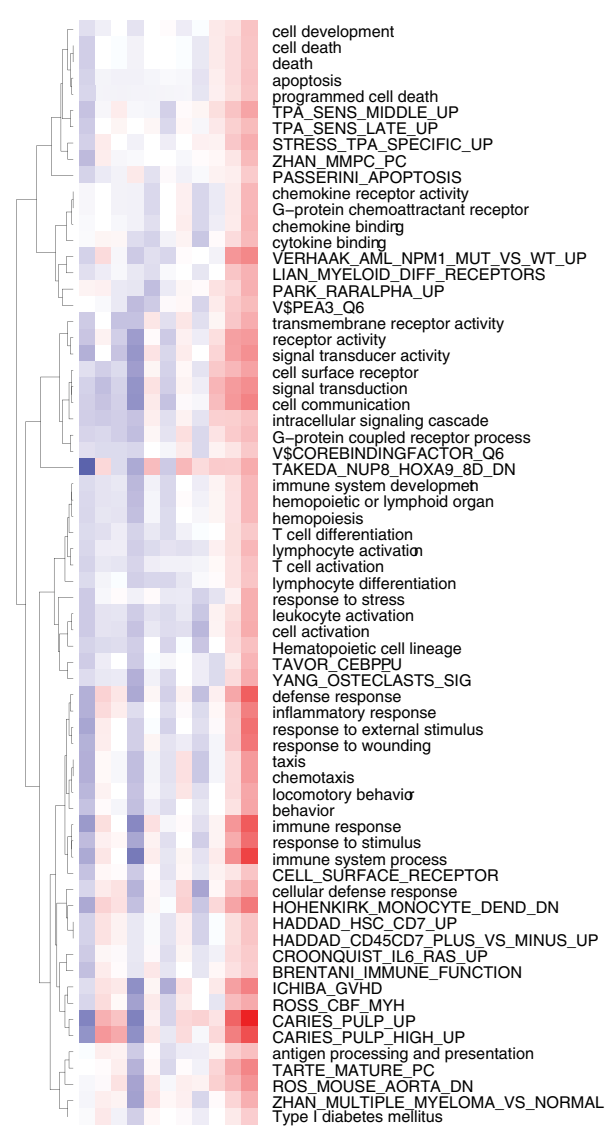

C

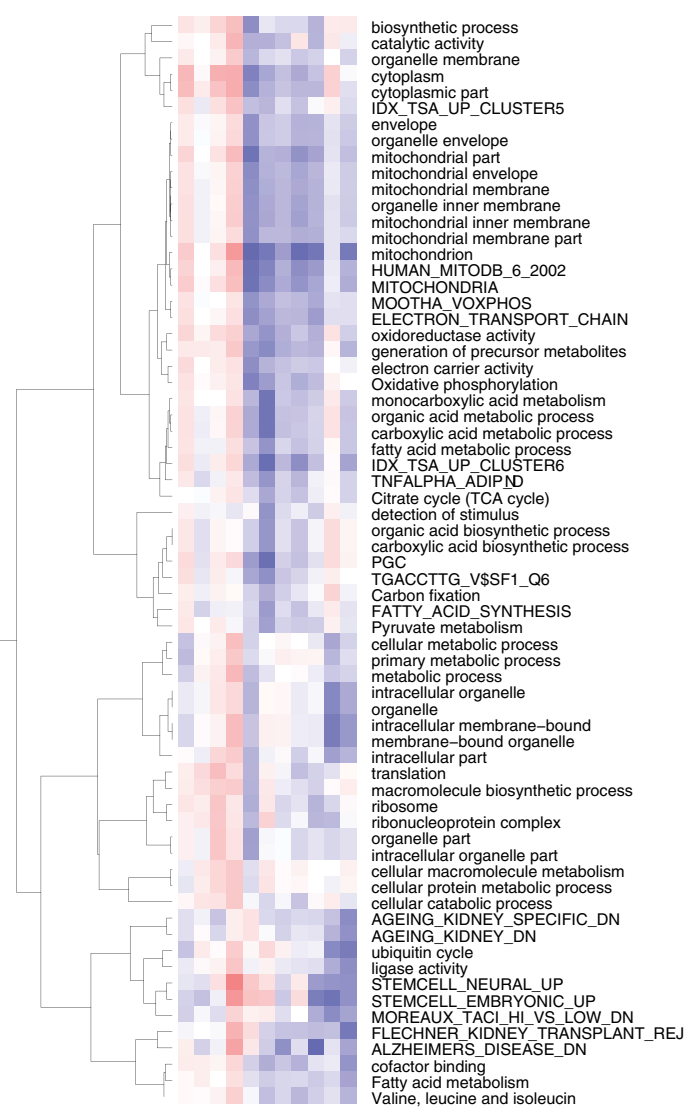

Fig. 2 Hierarchical clustering of (1) pathway's T-scores in MCT versus PUFA comparisons and (2) subjects according to their pathway activity profiles. a All pathways significantly $(E<0.05)$ differentially affected between two treatments in at least one of the subjects
( $n=547)$. b Sub-cluster containing apoptotic and immune pathways. c Sub-cluster containing mitochondrial and metabolic pathways. Color coding: Red positive T-values (up-regulation), Blue negative $\mathrm{T}$-values (down-regulation)
(Fig. 4a), while ADIPOQ MCT/PUFA expression ratio decreases in subjects with higher WHR (Fig. 4b). Therefore, the expression changes of these two genes reflect an increased inflammation of adipose tissue coupled with decreased expression of adiponectin in UBO subjects when MCT diet is consumed instead of the PUFA diet. Again, this suggests that MCT-rich diet may aggravate the obesity-related complications in subjects with high WHR (tendency to UBO). In contrast, this diet may have favorable effect for LBO subjects, as inflammation decreases and adiponectin increases in these subjects when they consume MCT instead of PUFA diet.

\section{Discussion}

Long and medium-chain fatty acids have different metabolic routing upon consumption: while long-chain fatty acids are considered to be disposed to the periphery, medium-chain fatty acids are rather disposed centrally. In addition, medium-chain fatty acids are more rapidly oxidized, increase energy expenditure after short-term feeding and attenuate weight gain after long-term feeding compared to long-chain fatty acids [10]. These differences in metabolic handling form a basis for a belief that consumption of MCT (as opposed to PUFA) may be beneficial for improving obesity-associated disorders.

In this study, we investigated the effect of body fat distribution on the physiological response to dietary fat interventions based on MCT and long-chain fatty acids (PUFA). The microarray gene expression profiling of the subcutaneous adipose tissue revealed that (1) there is a large variation in the transcription response to MCT and PUFA diets between UBO and LBO subjects, (2) UBO subjects are more sensitive to MCT versus PUFA dietary intervention than the LBO subjects and (3) the regulation of immune and apoptotic response and metabolic activity (including oxidative, lipid, carbohydrate and amino acid 

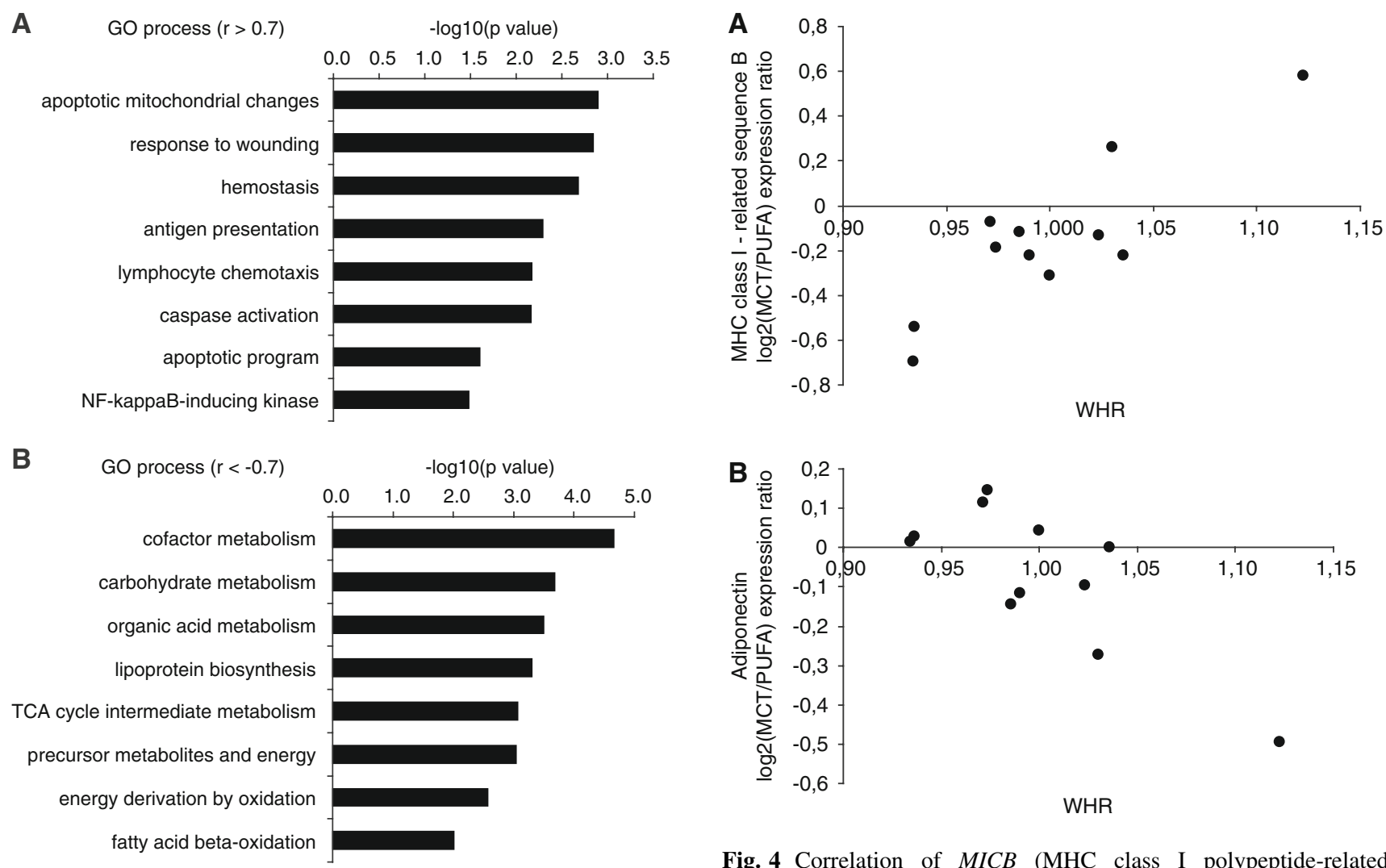

Fig. 3 Overrepresented GO categories among genes which expression is either positively or negatively correlated to WHR. a Overrepresented GO categories among genes with $\log _{2}$ (MCT/PUFA) ratios significantly positively correlated $(r>0.7, p<0.016)$ to WHR. b Overrepresented GO categories among genes with $\log _{2}$ (MCT/PUFA) expression ratios significantly negatively correlated $(r<-0.7, p<0.016)$ to WHR

metabolism) in response to MCT versus PUFA dietary intervention can be predicted by subject's body fat distribution: immune and apoptotic response increase, while metabolic activity decreases with increasing WHR.

These results demonstrate that the benefit of consumption of MCT-rich diet on obesity disorders strongly depends on body fat distribution. Although this diet may have positive effects for LBO subjects, increased inflammation and decreased energy metabolism suggest that MCT-rich diet has adverse effect on obesity-related complications of UBO subjects. The origin of these negative effects may be associated with hepatic fatty acid overload in UBO subjects. Since the UBO phenotype is usually associated with non-alcoholic fatty liver disease (NAFLD), transport of MCT directly to the liver by the portal vein may result in excessive hepatic fatty acid burden. This in turn would cause negative effects on obesity disorders on the systems level and provoke adverse changes that we detect in adipose tissue. The proposed mechanism is not plausible in LBO subjects because the particular risk of

Fig. 4 Correlation of $M I C B$ (MHC class I polypeptide-related sequence $\mathrm{B}$ ) and $A D I P O Q$ (adiponectin) gene expression ratios with WHR. MICB (MHC class I polypeptide-related sequence B) and $A D I P O Q$ (adiponectin) are examples of genes with $\log _{2}$ (MCT/PUFA) expression ratios significantly positively and negatively correlated ( $r=0.87, p=0.0005$ and $r=-0.78, p=0.004$, respectively) to WHR values in 11 subjects. MICB is stress-induced protein involved in immune response, while low adiponectin expression is an acknowledged marker for obesity-associated disorders. Subjects with increased WHR (upper body obesity) have induced expression of $M I C B$ and repressed expression of $A D I P O Q$ in their adipose tissues when consuming MCT versus PUFA diet

developing hepatic steatosis is specific to central obesity (UBO) [9].

The results obtained in this study highlight the importance of the personalized approach to understanding of a complex relationship between diet and physiology. The body fat distribution emerges as an important parameter to consider when assessing effects of long and medium chain dietary fats. Employment of a means to predict subjectspecific effects of dietary interventions, such as gene expression signatures and biomarker levels, may be an essential component necessary to provide an appropriate dietary recommendation.

Acknowledgments This study was financially supported by Dutch government through grant "Healthy nutrition" (Grant number 04003), Ministry of Health, Welfare and Sport, the Netherlands. Delegated sponsor of the study is BU Biosciences, TNO Quality of life, The Netherlands. 
Conflict-of-interest statement Authors declare not to have any conflict of interest.

Open Access This article is distributed under the terms of the Creative Commons Attribution Noncommercial License which permits any noncommercial use, distribution, and reproduction in any medium, provided the original author(s) and source are credited.

\section{References}

1. Biolo G, Toigo G, Guarnieri G (2001) Slower activation of insulin action in upper body obesity. Metabolism 50(1):19-23

2. Boorsma A, Foat BC, Vis D, Klis F, Bussemaker HJ (2005) T-profiler: scoring the activity of predefined groups of genes using gene expression data. Nucl Acids Res 33(Web Server issue):W592-W595

3. Cotter R, Taylor CA, Johnson R, Rowe WB (1987) A metabolic comparison of a pure long-chain triglyceride lipid emulsion (LCT) and various medium-chain triglyceride (MCT)-LCT combination emulsions in dogs. Am J Clin Nutr 45(5):927-939

4. DeLany JP, Windhauser MM, Champagne CM, Bray GA (2000) Differential oxidation of individual dietary fatty acids in humans. Am J Clin Nutr 72(4):905-911

5. de Hoon MJ, Imoto S, Nolan J, Miyano S (2004) Open source clustering software. Bioinformatics 20:1453-1454
6. Eisen MB, Spellman PT, Brown PO, Botstein D (1998) Cluster analysis and display of genome-wide expression patterns. Proc Natl Acad Sci USA 95:14863-14868

7. Krotkiewski M, Björntorp P, Sjöström L, Smith U (1983) Impact of obesity on metabolism in men and women. Importance of regional adipose tissue distribution. J Clin Invest 72(3):11501162

8. Metges CC, Wolfram G (1991) Medium- and long-chain triglycerides labeled with 13C: a comparison of oxidation after oral or parenteral administration in humans. J Nutr 121(1):31-36

9. Nugent C, Younossi ZM (2007) Evaluation and management of obesity-related nonalcoholic fatty liver disease. Nat Clin Practice Gastroenterol Hepatol 4(8):432-441

10. Papamandjaris AA, MacDougall DE, Jones PJ (1998) Medium chain fatty acid metabolism and energy expenditure: obesity treatment implications. Life Sci 62(14):1203-1215

11. Ramirez M, Amate L, Gil A (2001) Absorption and distribution of dietary fatty acids from different sources. Early Human Develop 65(Suppl 2):S95-S101

12. Reich M, Liefeld T, Gould J, Lerner J, Tamayo P, Mesirov JP (2006) GenePattern 2.0. Nat Genetics 38:500-501

13. Sanderson LM, De Groot PJ, Hooiveld GJEJ, Koppen A, Kalkhoven E, Müller M, Kersten S (2008) Effect of synthetic dietary triglycerides: a novel research paradigm for nutrigenomics. PLOS One, 3(2), art no e1681

14. St-Onge MP, Jones PJ (2002) Physiological effects of mediumchain triglycerides: potential agents in the prevention of obesity. J Nutr 132(3):329-332 